\title{
Humble Opinion on Okinawan Dietetic Culture in Pig's Retribution
}

\author{
DingYuebin \\ School of Foreign Languages, JiShou University, ZhangJiajie, China (dingyuebin@yeah.net)
}

\begin{abstract}
The paper focuses on the 115th Akutagawa Prize winning works Pig's Retribution, in which gives a special analysis of the Okinawan Dietetic culture and further to recover the Orientalism stigma hidden behind it.

Keywords-Matayoshi, Pig's Retribution, Okinawa, dietetic culture

\section{刍议又吉荣喜《猪的报应》的冲绳饮食文化}

丁跃斌

吉首大学外国语学院, 中国湖南省张家界市
\end{abstract}

摘要 本文以第 115 界芥川奖作品, 又吉荣喜的《猪的报应》为研究对象, 分析其作品中的冲绳的饮食文化, 进而揭示出隐匿其背 后的东方主义的特质。

关键词 又吉荣喜, 《猪的报应》, 冲绳, 饮食文化

\section{1. 引言}

2001 年《冲绳问题------世界最长寿民族的健康秘诀及 攻略》一书的出版, 使得冲绳传统的饮食文化受到了英语 国家的广泛追捧。该书作者美国内科专家和人类学家布雷 德利. 威尔科克斯和克雷格. 威尔科克斯兄弟亦变身为冲绳 人民的代言人, 将冲绳誉为人类的 “世外桃源”, 认为冲绳 饮食多以低热量的植物性饮食为主, 且饮食中未经提炼的 碳水化合物含量较高, 这不仅符合美国国家癌症研究所推 荐的食谱, 而且在某种程度上甚至超越了其他大多数科学 和医学权威所推崇的饮食标准。当然, 本文所探讨的重点 并不在于对上述相关科学资料的证实, 或是书中的引人注 目的言论, 亦或是对冲绳饮食文化的赞许; 我要强调的是 上述有些观点对冲绳文化意象起到了促进和被促进的作 用, 连西方人和日本本土人都对开始关注这种文化意象的 培养, 只不过他们在吸收这种文化的形式的过程中, 带有 一种复杂的情感, 即在赞美的同时又夹杂着些许屈尊的傲 慢。

在《冲绳饮食的影响力》一书中, 岸朝子也描绘了冲 绳的传统生活方式, 并指出在这些南部岛屿上存在着某些 优良的生活品质, 这种特质却在自利主义蔓延的现代日本 社会早已丧失。在尊重祖先的生活方式中, 在相互帮助的
仁慈精神中, 在节日和庆典时共享食物的慷慨习俗中, 岸 朝子发现了冲绳民众生活中的一些优良的古老传统。岸朝 子认为, 正是因为长期保留这些优良的古老传统, 才使得 冲绳岛民能够保持其本土文化的完整性, 远离超市、便利 店、加工和包装食品等没有任何优势的现代因素。

然而, 威尔科克斯兄弟也在暗示冲绳有多落后。在这 种矛盾情绪的纠缠下, 他们言论最初听起来像是善意的玩 笑, 但实际上, 如果深入理解就会发现隐匿在暗处的偏见。 他们的言论通常停留在主观固有的臆测上, 且对这种臆测 也从未质疑过, 因此在评论的主体和客体背后存在着强与 弱的二元对立。在一定程度上, 主体总是具有控制和支配 客体的权利, 所以威尔科克斯兄弟和岸朝子所作出的关于 冲绳的评论, 也是具有某些殖民特征的。受制于这种话语 权的叙述结构, 他们的言论不管设计的多么好, 终究无法 揭示其要表述的真实意图。这就是当我们试图描述那些我 们定义为政治和文化的“他者”时, 所通常面临的困境。当 把某些人描述成“他们”, 而不是“我们”的一部分时, 就会产 生麻烦的障碍, 阻止我们理解其真正要表达的意思。我们 理解问题的方式限定了我们解决问题的思路。众所周知, 爱德华. 萨义德提出了“东方主义”的概念, 用以描述西方学 者在接触东方文化、社会和种族的时候, 所表现出的那种 
思想矛盾和感情冲突。

\section{2. 吃}

《猪的报应》中作者运用大量的笔墨描绘各种“吃”的 场面。通常说来, 饮食的论述常常与文化习俗和社会价值 观紧密相连, 例如, 《冲绳饮食百科全书》中第三卷最后一 篇文章的作者认为“一个积极乐观的人, 永远懂得享受有效 的寿命与品味美食的密切关系。”与此同时, 也强调了因为 良好的饮食习惯而形成的在家庭中男女之间相互扶持的重 要性, 即, 妻子在家庭中负责食物的㙜饪, 而其他的家庭 成员则负责来品尝餐桌上的食物。或者说是母亲承担做饭 的工作, 而父亲和孩子则充分的享受这些美食, 这种理想 化的家庭分工观念已经成为资产阶级核心家庭的标准模 式。另外, 《冲绳饮食百科全书》还深入的肯定了在这种理 想化的家庭模式变为现实的过程中丈夫的理解和辅助起着 不可或缺的作用。

通读《猪的报应》可以发现在小说中这种现代家庭模 式被完全颠覆, 作品中无数个关于“吃” 的场景都无一例外 的证明了这一点。例如, 小说中的三位女主人公美代、畅 子及和歌子她们都是自己做饭自己吃, 而且她们奇特的食 欲似乎也暗示着她们对理想的资产阶级家庭模式所做出的 绝望性的报复。她们无法融入这种家庭模式, 就婚姻而论, 她们很显然都是失败者。

现在我们来探讨一个根本性的问题, “什么是吃? ”, 也就是, 进食的寓意是什么? 马吉 - 基尔格认为进食是一种 合作形式。它包括两个相对立的功能: 异化和同化。在异 化功能中, 进食涉及几对界限分明的术语一一食者和食物, 胜利者和受害者, 强者和弱者等。他们彼此间有着清晰的 界限, 且不平等, 更不用说是互惠互利了。前者总是试图 压制和掌控后者。然后, 在同化功能中, 上述权利结构则 被彻底颠覆。界限分明的几对术语变得不那么容易区分, 甚至出现逆向倾向。在进食过程中, 食者通过吞食食物维 持生存, 换言之, 食者需要依赖食物才得以活下去, 在某 种程度上, 也就是与 “他者”合二为一。如此看来, 同化和 异化两种对立的功能在进食过程中要达到健康的平衡点, 就需要借助一种相对理想化的消耗方式, 马吉 - 基尔格把这 种方式成为 “共同参与”。也就是说, 这种平衡在很大程度 上需要食者和食物的和谐共存, 食者必须确保不能耗尽某 类食物, 只要后者不对其不产生威胁的情况下, 允许它加 以成长。食者与食物的关系就如同胜利者与受害者, 强者 与弱者一样需要依赖在同化和异化过程中那细微平衡才能 得以维持。

在小说中, 有一个情节需要我们特别留意。正吉警告 三个女主人公不要告诉岛民她们来真谢岛的真正目的, 并
解释说“这些岛民不愿意外来人朝拜御岳, 他们认为外人无 权向岛上的众神祈愿。”畅子听后开玩笑的认为岛民的谨慎 是因为和歌子太贪婪了。她说“因为和歌子是贪心的人, 他 们觉得和歌子会把岛上的幸福全部拿走的。”之后畅子还指 责她的同伴和歌子, “在酒吧里, 哪怕是客人的东西也想 要。”听到这些后, 她们的老板美代不但不制止她们的争论, 反倒还帮腔地说“如果被和歌子拿走的话, 就什么也没有 了, 也许岛上的全部幸福只够和歌子一个人拿的吧。”

虽然这三个女人并未意识到这一点, 但是, 她们提到 的正是基尔格所提出的同化和异化间的细微的平衡。真谢 岛的“幸福”无疑包含其文化、经济环境及礼仪习俗, 甚至 直指这个小岛的全部生活。为了抵制来自外部的社会文化 压力, 他们不得不通过维持这个小环境里的食者与食物间 细微的平衡来保护他们的“幸福”。当他们试图收割植物或 是猎杀动物时, 同时也会遭到他们所伤害的物种的反击报 复, 那么岛上的生活就无法维持原状。这三个女人对成为 这个脆弱生存空间 (真谢岛) 的入侵者, 竟然不以为然, 甚至认为即使岛上的“所有幸福”因她们而消失, 她们也不 愿承担一点责任。因为即使岛上的幸福都被她们拿走了, 也是因为岛上的幸福本来就没多少。基尔格所要告诫的就 是这种形式的贪婪，他的“共同参与理论”指向的是一种健 康的合作形式, 是同化和异化的和谐共生。他认为一旦这 种健康的平衡被打破，那么合作的意愿就会办成赤裸裸的 毁灭, 甚至变成嗜食同类, 相互杀翏而食。“共同参与”意 图联合“他者”，在健康的环境下共同成长；而“嗜食同类” 则是受那种不消灭他者就会被消灭的毁灭性的欲望所操 控。

然而, 基尔格承认, 前者也存在变为后者的潜在危险。 恋爱的结局最后往往就会睢入贪婪的性欲, 火热的情感最 后变为冷酷的性侵。事实上, 又吉荣喜《猪的报应》就灌 输着可怕的“嗜食同类”的主题。

\section{3. 食人}

仔细研究人类学关于“食人”的研究成果, 就会发现一 个有趣的现象。人类学家阿伦斯认为到目前为止没有完全 直接的证据可以证明人类社会曾经出现过食人现象, 所有 关于食人的记载都是传闻。将所有资料进行整理, 事实就 变得清晰了, 阿伦斯发现, 几乎所有关于“食人”的记载都 是属于那些居住在没有任何通讯联系的偏远地区的人们, 或是那些在神话中被描述为早期部落时代的远祖。对某个 共同体的成员来说这些人很可能被看成“他者”。那些把他 们认定为食人者的结论简直荒唐透顶。鉴于此, 阿伦斯提 出不能将“食人”看成是普通的现实, 而是作为一种区分文 明国度和野蛮国度的隐性标志。人们总是试图通过强调自 
己与那些野蛮人的不同来证明自己有多文明。阿伦斯指出 当人们将矛头指向 “他者”时, 就会采取上述行为, 并把那 些“他者”称作“食人者”。

阿伦斯认为“某个群体对“他者”的食人本性的臆断可以 解释为文化边界构建与维系的一个方面”。”简言之, 人们 常常用“在我们的国土之外存在着残忍的食人族。”这样的 话来炫耀他们自己是多么文明。也就是说, “他们是食人族” 这样的谴责本身, 并不能证明任何相关的事实的存在, 而 只是仅仅揭示出谴责者对被谴责者的强烈的歧视。这种深 刻的见解不仅适用于宗族歧视, 同样适用于全部种族歧视 和女性歧视。事实上, 大量的记载也只是在指责那些被认 为是食人族的社会劣势。因此, 殖民者总是把那些造反的 原住民叫做食人族, 甚至在原始的父权社会, 当男人离开 后, 他们就会怀疑女人和孩子会以人肉为生。阿伦斯指出 几乎所有的关于食人的记载都宣扬这种该死的食人的习性 是属于那些没什么学识修养, 只有蛮力的人。现在, 当我 们了解食人所具有的象征意义后, 我们就能轻易的理解这 背后的原因。

说完这些, 让我们回到《猪的报应》, 有趣的是, 在故 事的绝大多数进食场景中, 我们可以发现, 都是女人, 在 享受美食后又遭受其折磨, 而男性几乎没有。在进食过程 中, 对女性脸部的描写, 特别是对其嘴部的描写, 被不可 思议地夸大, 甚至有些色情。

在故事最初的一个场景里, 美代在一个小港口等待开 往真谢岛的渡船, 她的香草冰淇淋因为融合掉色的口红而 变得微红, 她微厚的嘴唇也因退了色而显得格外柔弱。另 一个场景里, 描绘了引发后来食物中毒的晚宴。民宿的老 板为了感谢她们把酒醉的老板娘送到诊所, 送了他们一份 用黑色塑料袋装着的食物, 里面有“黑的, 红的, 长的, 软 乎乎的, 很多样”。正吉发现 “女人们的嘴湿漉漉的, 大口 吃猪肉, 大口喝泡盛酒”。当畅子与和歌子刚刚从食物中毒 中恢复过来, 就开始喝鱼汤, 红色的口红沾了油, 泛着光。 同样有趣的场景还有美代、民宿的老板娘和护士在诊所一 起喝墨鱼汤, 嘴上都变得黑乎乎的。小说中确实描写了很 多关于女人进食的场景。

其中, 在岛上第二天的猪肉晚宴需要特别提及, 而且 这个晚宴还引发了集体食物中毒。非常有趣的是, 和歌子 把这顿晚宴叫做“最后的晚餐”。

女人们的嘴湿漉漉的, 她们大口吃猪肉, 大口喝泡盛。 紧接着, 话变得越来越多, 大笑声此起彼伏。令正吉不可 思议的是这么苗条的身材竟然这样能吃能喝。猪肋骨、猪 蹄骨在盘子上越堆越高, 最后从盘子上滑下, 散落在矮脚 桌上。汤已经凉了, 油淢淢地漂着灰白的猪油, 但是女人
们照样伸着脖子大口喝着。畅子不时地把手指伸进杯子里 的泡盛, 摚动着里面的冰块。美代像是吸进去似的把肉吞 到嘴里, 然后闭上嘴, 腮帮子一鼓一鼓地, 然后灵巧地把 白乎乎的骨头吐出来。

小说中用这种充满诙谐和怪异的进食场景来描述女人 们被压抑的性欲, 同时也引出了集体中毒这个次要情节。 上面的引文也象征性的体现了对女性的歧视。有趣的是, 正吉, 这个晚宴中唯一的男性, 背地里却在担心, 他害怕 女人们会因为他只是吃了一些海带和夢卜而觉得扫兴。这 一段主要强调不情愿的旁观者的社会文化远远区别于急不 可耐的食人族。最后因为晚宴引发的食物中毒则作为一个 对野蛮女人们的象征性的制裁。

最具重要意义的是, “最后的晚餐”的整个场景都充斥 着“食人”和“性交”的意象。

首先, 当女人们谈论猪将自己而献给他人做食物有多 高尚时, 食者与食物间的象征性的差异就被瞬间转移, 谈 论的主题从“进食”跨越至“性”。至此为止, 她们谈论的主题 似乎变成了对基于相互奉献的男女之间的理想伴侣关系的 渴求上。

而事实并非如此。当和歌子告诉他人”我们必须全部吃 完, 一滴都不能剩”时, 对话的主题突然改变。其中“一滴 都不能剩”也在暗示着对男人的精液的贪婪。与此同时, 食 者与食物间的差异性被重新确定, 女性对怀孕问题的理解 则突现其在性上的主导地位。

如果猪神真的存在, 也是对女人有利的, 可以彻底地 满足她们的性欲和食欲。美代说“我还是觉得无论怎样折腾 都不会怀孕为好. ”很明显地证明了她的自私。“怀孕”不仅 暗示着资产阶级潜意识中所追寻的“幸福”, 而且含蓄地要 求女性对其性行为负全责。美代, 一个性欲很强的自私女 人, 当然认为怀孕是一种非理想化的状态。其女色情狂的 本性可以从畅子的问题和美代对正吉的告白中可管窥一 二。畅子曾经问美代“你拒绝过男人吗? ”可见, 美代对男 性的欲望使得她可以接受任何男人, 所以自然不会轻易回 绝男人。美代自己也默认她曾对正吉说“正吉, 只要看看你 肩膀也会勾起我的性欲”。和歌子说 “要是女的, 我们吃掉 它们就成自相残杀了。”这句话掩饰了她对争夺玩弄男性的 厌倦, 害怕最后会使她们因为男人而内讧。“女人们的眼睛 亮闪闪的发光, 嘴唇上的口红的颜色没有了, 粘着油脂发 着暗光。”事实上, 这三个女人美代、畅子及和歌子她们为 了争夺同行中唯一的男性正吉而彼此进行着竞争。因此, 在“最后晚餐”这一场景中, 有效的运用了“食人”的主题, 来 描述女人们对“幸福”和“性”的渴望。 


\section{参考文献(References)}

[1] Arens,W.The Man-Eating Myth: Anthropology and Anthropophagy. New York: Oxford UP,1979

[2] Kilgour, Maggie. From Communion to Cannibalism:An Anatomy of Metaphor of Incorporation. Princeton: Princetion UP, 1990.
[3] Said, Edward W. Orientalism. Vintage Books, 1979.

[4] Willcox, BradleyJ., D.Craig Willcom, and Makoto Suzuki. The Okinawa Program: How the World'sLongest-Lived People Achieve Everlasting Health and How You Can Too. New York: ClarksonPotter publishers, 2001

[5] Matayoshi .Pig's Retribution. Tokyo: Bungeishunju Ltd, 1996. 